\title{
Cell membrane-derived biomimetic nanodecoys for viruses
}

\author{
Xuan Liu, Dengfeng Li \& Gang Liu* \\ State Key Laboratory of Molecular Vaccinology and Molecular Diagnostics \& Center for Molecular Imaging and Translational Medicine, \\ School of Public Health, Xiamen University, Xiamen 361102, China
}

Received February 24, 2020; accepted February 27, 2020; published online May 12, 2020

Citation: Liu, X., Li, D., and Liu, G. (2020). Cell membrane-derived biomimetic nanodecoys for viruses. Sci China Life Sci 63, 1254-1256. https://doi.org/ $10.1007 / \mathrm{s} 11427-020-1669-\mathrm{x}$

Viruses cause numerous acute, chronic and life-threatening infectious diseases, and remain a major public health problem worldwide. The wide-spreading and highly pathogenic viruses, including hepatitis virus, human immune deficiency virus (HIV), Zika virus (ZIKV), influenza and Ebola, bring huge medical burden (Guo et al., 2019; Holmes et al., 2016). Currently, there are 250 million chronic HBV carriers (Yuen et al., 2018) and 37.9 million HIV carriers worldwide (WHO, 2018, https://www.who.int/hiv/data/en/). As a global killer, seasonal epidemics of influenza could result in about 3 to 5 million cases of severe illness and 200,000 to 650,000 deaths annually (Woyessa et al., 2018). Up to date, SARS-CoV-2 has caused over two million cases in more than 213 countries, areas or territories with over 150,000 deaths (WHO, 2020, https://www.who.int/emergencies/diseases/novel-coronavirus-2019/situation-reports/). Although anti-pathogenic agents and vaccines were introduced into modern medicine in the last century, an estimated $25 \%$ of annual deaths worldwide are directly attributed to infectious diseases (Morens et al., 2004). These epidemiological data signal a clear urgency and highlight the importance of developing anti-infection approaches to address the resistance or deficiencies of antiviral formulations and vaccines (Bricarello et al., 2012). For most viruses, interaction with attachment factors and receptors expressed on the host cell membrane is an essential process to establish infection (Marsh and Helenius, 2006). Such pathogenic binding and entry mechanisms offer opportunities for developing broadly applicable anti-

*Corresponding author (email: gangliu.cmitm@xmu.edu.cn) viral strategy, which subvert the interaction of specific membrane proteins and viruses.

In recent years, cellular membrane vesicles (MVs)-based therapeutics have been extensively investigated as delivery systems for drugs or imaging contrast agents, as a result of improved tolerability, targeting and circulation, as well as their capacity to communicate with cells via signal transduction and membrane fusion (Shi et al., 2020; Zhang et al., 2020). Rather than an artificial membrane synthesized from fully synthetic components, MVs are best owned natural properties of the source cell membrane in a straight forward manner. The laterally mobile, two-dimensional lipid-bilayer environment and specific molecular compositions play an important role in modulating the conformational equilibria of proteins and coordinating the bioactivity of membrane constituents (Lingwood and Simons, 2010). For early interruption of virus-to-host interactions, MVs that present highspecificity mimics of cellular receptors may provide potential alternative exogenous decoys to trick viruses (Figure $1 \mathrm{~A})$.

For viral infection inhibition, MVs mimicking cell-surface receptors could compete with native cellular receptors to bind to the specific ligands. For example, Wei et al. (Wei et al., 2018) reported a nanoparticle-based broad-spectrum antivirus strategy through fusing lymphocyte cell membranes onto polymeric cores, which makes them ideal decoys of natural $\mathrm{CD}^{+} \mathrm{T}$ lymphocyte cells. The resulting T-cellmembrane-coated nanoparticles (TNPs) inherit T cell surface antigens critical for human immunodeficiency virus (HIV) binding, such as CD4 receptor and CCR5 or CXCR4 co- 


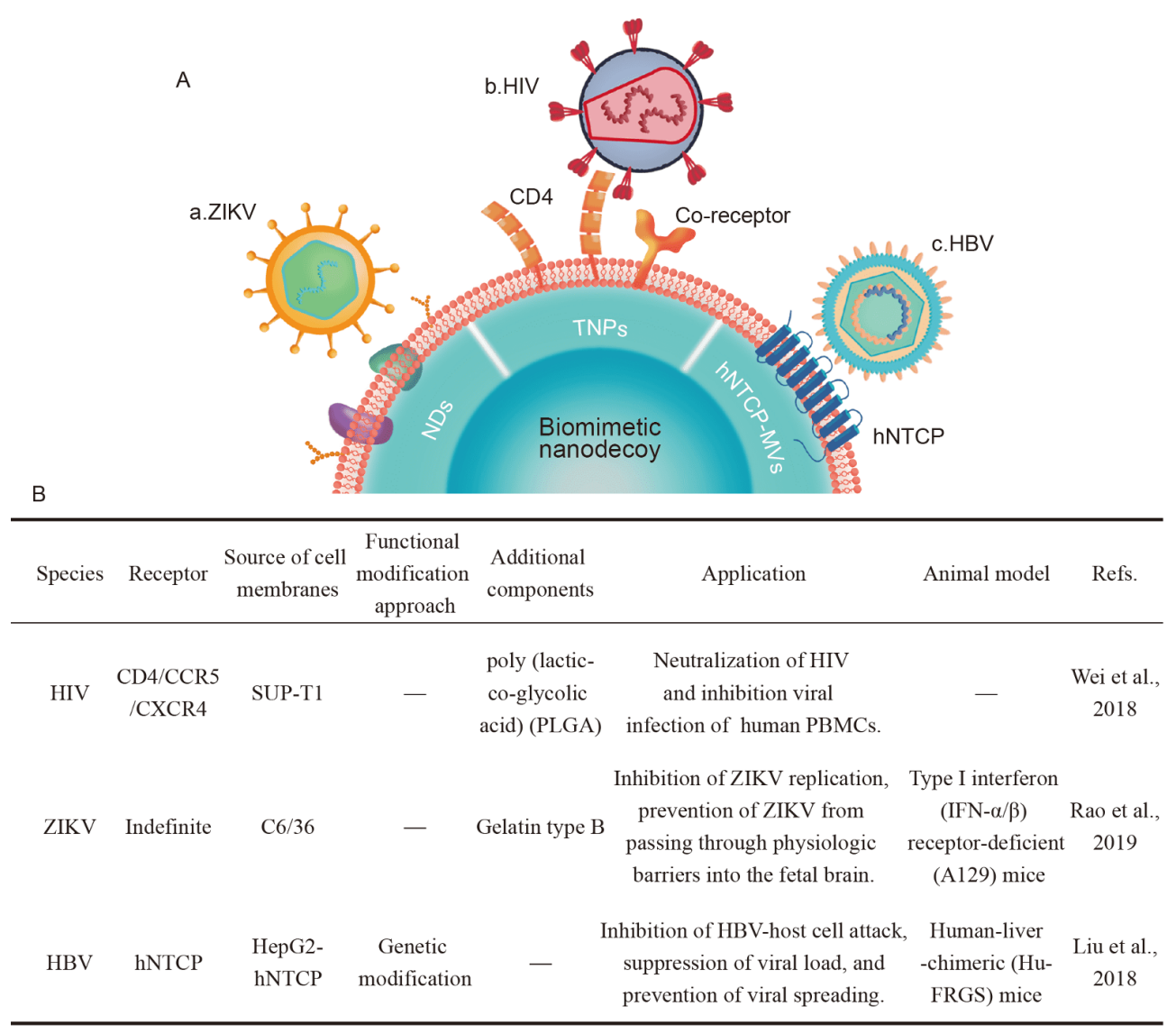

Figure 1 (Color online) Cell membrane-derived biomimetic nanodecoys (A) and summary of typical biomimetic nanodecoys (B). a, Zika virus (ZIKV); b, human immunodeficiency virus (HIV); c, hepatitis B virus (HBV).

receptors. Through selectively binding with gp120, a key envelope glycoprotein of HIV, TNPs effectively neutralized HIV and inhibited viral infection of human peripheral mononuclear blood and human-monocyte-derived macrophages. For viruses whose receptors are not clear, natural host cells or medium host cells are available membrane source. To divert Zika virus (ZIKV) away from its intended targets, an anti-ZIKV host-mimicking nanodecoy (ND) constructed by wrapping a polymeric core with mosquito medium host cell membranes was developed (Rao et al., 2019). The NDs could significantly prevent ZIKV replication in vitro, negate ZIKV-induced inflammatory and degenerative changes, and improve the survival rate in mouse models. What is perhaps most convenient about these cell membrane-based viral decoys is the natural binding ability of host cells that makes the exogenous engineering of receptor moieties unnecessary.

However, in many cases, the host cells of some viruses are too special to obtain easily. For example, human hepatitis B virus (HBV), which is closely related with liver cirrhosis and hepatocellular carcinoma (HCC), only attacks primary hepatocytes of humans, chimpanzees or Tupaia generally (Yuan et al., 2019a; Yuan et al., 2019b). Key challenges in the use of these primary cells for infection inhibition include difficulties in their harvesting and preservation of their integrity. Rather than employing natural cell membrane directly, cell plasma membrane can further be engineered artificially to display more active proteins and thereby be endowed with specific virus-binding components. However, most cell membrane vesicles, which are chemically engineered with functional motifs, often suffer from poor biological stability and are limited in terms of in vivo delivery logistics and therapeutic activity (Dehaini et al., 2016).

To address the limitations of receptor-mediated HBV antiviral treatment and functional modification, HBV-specific receptor, a nine-transmembrane transporter named sodium taurocholate co-transporting polypeptide (hNTCP), was naturally immobilized onto the cellular surface via a genetic modification procedure (Liu et al., 2018). Membrane vesicles derived from the modified cells, named hNTCP-MVs, were conferred important surface properties to play a specific biological function. The hNTCP-MV could recognize and bind to the dissociative HBV virion with high specificity and sensitivity in vitro due to endogenous display of hNTCP. The treatment of hNTCP-MV could prevent viral infection, spreading, and replication effectively in human liver chimeric mice, an experimental mouse model of HBV infection. The curative therapeutic outcome indicated promising po- 
tential for future clinical translation and this work opens up new perspectives for biomimetic binding strategies as valuable tools to overcome the clinical challenges against virus infection.

Infectious diseases have caused incalculable misery and death. The diversity of infectious disease threats currently facing humanity is unprecedented. However, the complex mechanism and progress of diseases induced by many viruses have still not been fully known, nor did a functional cure was achieved. Nevertheless, suppression of viral infection and spreading may be the central role to prevent the progress of virus-induced diseases, especially in the cases of virus outbreak that lack antiviral drugs and vaccines. As an intersection of nanotechnology and biomedical technology (Chen and Liang, 2018), nanomedicine created novel solutions for addressing some of the most pressing human health needs (Liu et al., 2020; Wang et al., 2019; Zhang et al., 2018). By taking inspiration from natural host cell biological systems, MVs acted as an outstanding nano platform for functional moieties anchoring. These nano "decoys" that present high-specificity mimics of receptors can hinder viral attachment and inhibit cell entry process. Due to the varied sources from host and bioengineering cells, MVs can be exploited to target the vast viral repertoire, and be used as a broad-spectrum platform in antiviral therapy (Figure 1B).

Moreover, as a biomimetic delivery system, another promising feature of MVs is that MVs are flexible in customizability due to a myriad choices of loading cargos, including antiviral drugs, immunomodulatory agents and contrast agents/labeling probes. Moving forward, the complex of "decoy" and virus due to the receptor-virus affinity may enhance the presentation of viral antigen protein and elicit functional immunity capable of combating virus infections, which underscores the potential utility of biomimetic nanodecoy-based immunotherapy. It is believed that this customizable platform has great potential to be applied against a broad range of viruses efficiently and flexibly.

Compliance and ethics The author(s) declare that they have no conflict of interest.

Acknowledgements This work was supported by the Major State Basic Research Development Program of China (2017YFA0205201), the National Natural Science Foundation of China (81901876, 81422023 and U1705281), the Fundamental Research Funds for the Central Universities (20720190088 and 20720200019), and the Program for New Century Excellent Talents in University, China (NCET-13-0502).

\section{References}

Bricarello, D.A., Patel, M.A., and Parikh, A.N. (2012). Inhibiting hostpathogen interactions using membrane-based nanostructures. Trends
Biotech 30, 323-330.

Chen, S., and Liang, X.J. (2018). Nanobiotechnology and nanomedicine: small change brings big difference. Sci China Life Sci 61, 371-372.

Dehaini, D., Fang, R.H., and Zhang, L. (2016). Biomimetic strategies for targeted nanoparticle delivery. Bioeng Translational Med 1, 30-46.

Guo, H., Cai, C., Wang, B., Zhuo, F., Jiang, R., Wang, N., Li, B., Zhang, W., Zhu, Y., Fan, Y., et al. (2019). Novel hepacivirus in Asian house shrew, China. Sci China Life Sci 62, 701-704.

Holmes, E.C., Dudas, G., Rambaut, A., and Andersen, K.G. (2016). The evolution of Ebola virus: Insights from the 2013-2016 epidemic. Nature 538, 193-200.

Lingwood, D., and Simons, K. (2010). Lipid rafts as a membraneorganizing principle. Science 327, 46-50.

Liu, H., Yang, Y., Liu, Y., Pan, J., Wang, J., Man, F., Zhang, W., and Liu, G. (2020). Melanin-like nanomaterials for advanced biomedical applications: a versatile platform with extraordinary promise. Adv Sci 7, 1903129.

Liu, X., Yuan, L., Zhang, L., Mu, Y., Li, X., Liu, C., Lv, P., Zhang, Y., Cheng, T., Yuan, Q., et al. (2018). Bioinspired artificial nanodecoys for hepatitis B virus. Angew Chem Int Ed 57, 12499-12503.

Marsh, M., and Helenius, A. (2006). Virus entry: open sesame. Cell 124, 729-740.

Morens, D.M., Folkers, G.K., and Fauci, A.S. (2004). The challenge of emerging and re-emerging infectious diseases. Nature 430, 242249.

Rao, L., Wang, W., Meng, Q.F., Tian, M., Cai, B., Wang, Y., Li, A., Zan, M., Xiao, F., Bu, L.L., et al. (2019). A biomimetic nanodecoy traps Zika virus to prevent viral infection and fetal microcephaly development. Nano Lett 19, 2215-2222.

Shi, Y., Xie, F., Rao, P., Qian, H., Chen, R., Chen, H., Li, D., Mu, D., Zhang, L., Lv, P., et al. (2020). TRAIL-expressing cell membrane nanovesicles as an anti-inflammatory platform for rheumatoid arthritis therapy. J Control Release 320, 304-313.

Wang, J., Wang, Y., Wang, R., Zhang, S., Liu, X., and Liang, X.J. (2019). Targeted nanoparticles for precise cancer therapy. Sci China Life Sci 62, $1392-1395$.

Wei, X., Zhang, G., Ran, D., Krishnan, N., Fang, R.H., Gao, W., Spector, S. A., and Zhang, L. (2018). T-cell-mimicking nanoparticles can neutralize HIV infectivity. Adv Mater 30, 1802233.

Woyessa, A.B., Mengesha, M., Belay, D., Tayachew, A., Ayele, W., Beyene, B., Kassa, W., Zemelak, E., Demissie, G., Amare, B., et al. (2018). Epidemiology of influenza in Ethiopia: findings from influenza sentinel surveillance and respiratory infection outbreak investigations, 2009-2015. BMC Infect Dis 18, 449.

Yuan, L., Jiang, J., Liu, X., Zhang, Y., Zhang, L., Xin, J., Wu, K., Li, X., Cao, J., Guo, X., et al. (2019a). HBV infection-induced liver cirrhosis development in dual-humanised mice with human bone mesenchymal stem cell transplantation. Gut 68, 2044-2056.

Yuan, L., Zhang, Y., Liu, X., Chen, Y., Zhang, L., Cao, J., Li, X., Wang, M., Wu, K., Zhang, J., et al. (2019b). Agonist c-Met monoclonal antibody augments the proliferation of hiPSC-derived hepatocyte-like cells and improves cell transplantation therapy for liver failure in mice. Theranostics 9, 2115-2128.

Yuen, M.F., Chen, D.S., Dusheiko, G.M., Janssen, H.L.A., Lau, D.T.Y., Locarnini, S.A., Peters, M.G., and Lai, C.L. (2018). Hepatitis B virus infection. Nat Rev Dis Primers 4, 18035.

Zhang, X., Zhang, Y., Zhang, Y., Lv, P., Zhang, P., Chu, C., Mao, J., Wang, X., Li, W., and Liu, G. (2020). Bio-engineered cell membrane nanovesicles as precision theranostics for perihilar cholangiocarcinoma. Biomater Sci 8, 1575-1579.

Zhang, Y., Yu, J., Qiang, L., and Gu, Z. (2018). Nanomedicine for obesity treatment. Sci China Life Sci 61, 373-379. 